\title{
Wirkung von Einflussstrategien auf das Verhalten von Mitgliedern am Beispiel von Winzergenossenschaften
}

\author{
Max Härle und Jon H. Hanf \\ Hochschule Geisenheim University, 65366 Geisenheim, Germany
}

\begin{abstract}
Seit über 20 Jahren ist der deutsche Weinmarkt gesättigt mit dem Ergebnis, dass dort eine hohe Wettbewerbsintensität herrscht. Auf diesem Verdrängungsmarkt konkurrieren die Genossenschaften mit den großen Abfüllern und Weingütern um die wenigen Plätze im Lebensmitteleinzelhandel und Discount. Somit sind die die Händler in der Lage Qualität und Quantität klar vorzugeben. Folglich bestehen die Herausforderungen für das Management von Primärgenossenschaften auf der einen Seite in der Bereitstellung großer qualitativ hochwertiger und homogener Weinmengen und auf der anderen Seite in der Berücksichtigung der Auswirkungen ihres Handelns auf die Mitgliederbeziehung. In diesem Kontext ist das Ziel dieses Artikels zu untersuchen, welche Einflussstrategien zur Produktion von Qualitätswein sich für Geschäftsführer von Genossenschaften anbieten, um ihre Lieferanten bzw. Eigentümer bestmöglich zu koordinieren.
\end{abstract}

For over 20 years the German wine market is saturated with the result that there exists a high level of competition. In this competitive market, cooperatives compete with the big bottlers and wineries for the scarce places in the food retail and discount shelves. Thus, the retailers are able to specify the quality and quantity they want to have. Consequently, the challenges for the management of primary cooperatives are on one side to provide good quality, homogeneous quantities of wine and on the other hand to coordinate the impact of these specifications on their member businesses. In this context, the aim of this article is to investigate which influence strategies are best ones for the production of quality wines.

Depuis plus de 20 ans, le marché du vin allemande est saturé avec le résultat qu'il existe un niveau élevé de concurrence. Dans ce marché concurrentiel, les coopératives en concurrence avec les grands embouteilleurs et les caves autour des rares endroits dans le commerce de détail alimentaire et de réduction. Ainsi, les commerçants sont la qualité et la quantité clairement pour spécifier l'emplacement. Par conséquent, les défis pour la gestion des coopératives primaires existent d'un côté à fournir une grande qualité et des quantités homogènes de vin et d'autre part, à la lumière de l'impact de leurs actions sur la relation Membres. Dans ce contexte, l'objectif de cet article est d'enquêter sur qui offrent des stratégies d'influence pour la production de vins de qualité pour la gestion des coopératives, de coordonner leurs fournisseurs et les propriétaires que possible.

\section{Einleitung}

Seit über 20 Jahren ist der deutsche Weinmarkt gesättigt mit dem Ergebnis, dass dort eine hohe Wettbewerbsintensität herrscht. Auf diesem Verdrängungsmarkt konkurrieren die Genossenschaften mit den großen Abfüllern und Weingütern um die wenigen Plätze im Lebensmitteleinzelhandel und Discount. Somit sind die die Händler in der Lage Qualität und Quantität klar vorzugeben (Hanf und Schweickert 2014). Folglich bestehen die Herausforderungen für das Management von Primärgenossenschaften auf der einen Seite in der Bereitstellung großer qualitativ hochwertiger und homogener Weinmengen und auf der anderen Seite in der Berücksichtigung der Auswirkungen ihres Handelns auf die Mitgliederbeziehung. Hierbei ist zu berücksichtigen, dass bei den Mitgliedern zwischen wenigen großen Traubenproduzenten und vielen nebenberuflichen kleine Traubenproduzenten zu unterscheiden ist. Somit muss die Genossenschaft sehr heterogene Lieferanten, die gleichzeitig die Eigentümer darstellen, managen.
Aufgrund der Genossenschaft inhärenten EigentümerLieferanten Strukturen muss die Unternehmensführung einen Schwerpunkt ihrer Tätigkeit auf die Ausgestaltung der Kooperation mit ihren Lieferanten legen, da diese Lieferanten als gleichzeitige Eigentümer Einfluss auf die Unternehmenspolitik nehmen und im Extremfall über die Weiterbeschäftigung des Geschäftsführung entscheiden können. Neben den Herausforderungen, die aufgrund der Eigentumsstruktur resultieren, entstehen aufgrund der genossenschaftlichen Prinzipien weitere Herausforderungen. In diesem Kontext verweisen Cook und Iliopoulos (1999) auf Trittbrettfahrer-Probleme, PortfolioProbleme, Horizont-Probleme, Kontroll-Probleme und Einfluss-Probleme.

Viele der genannten Probleme von Genossenschaften findet man in der Supply-Chain-Literatur als Kooperationsprobleme (Hanf und Dautzenberg, 2006; Gulati et al., 2005). Laut Preckel et al. (2004) gibt es im Agri-Food-Business Supply Chains, die aus einem einzelnen Verarbeiter und vielen Zulieferern von Rohmaterial bestehen. Die Kooperation von Mitgliedern

This is an Open Access article distributed under the terms of the Creative Commons Attribution License 4.0, which permits unrestricted use, distribution, and reproduction in any medium, provided the original work is properly cited. 
der Supply Chain gilt ebenfalls als Voraussetzung für ein effektives Supply Chain Management (Ellram und Cooper, 1990). Kooperationsprobleme entstehen durch Interessenkonflikte und können sich negativ auf die Motivation auswirken (Gulati et al., 2005). Die Leistung eines Konstrukts hängt davon $a b$, wie gut die Partner miteinander kooperieren (Halldorsson et al., 2007), da die Möglichkeiten nicht voll ausgeschöpft werden, wenn die Mitglieder ihre eigenen Ziele in den Vordergrund stellen anstatt ihre Ziele und Aktivitäten zu koordinieren um das Gesamtergebnis der Kette zu optimieren (Cooper et al., 1997). Im Bereich des Supply Chain Mangements konnte im Kontext von Einflussstrategien gezeigt werden, wie vertikale Lieferketten aus Netzwerksicht optimal gesteuert werden können.

Ziel dieses Artikels ist es zu untersuchen, welche Einflussstrategien zur Produktion von Qualitätswein sich für Geschäftsführer von Genossenschaften anbieten, um ihre Lieferanten bzw. Eigentümer bestmöglich zu koordinieren. Anhand eines Beispiels wird gezeigt, welche Möglichkeiten sich der Geschäftsführung einer Genossenschaft durch die Zusammenarbeit mit externen Personen eröffnen. Hierfür werden wir im folgenden Kapitel kurz auf die Problematik von Winzergenossenschaften bei der Produktion von Qualitätswein eingehen. Im darauffolgenden Kapitel stellen wir Einflussstrategien dar und zeigen deren Bedeutung für das Supply Chain Management auf. Danach diskutieren wir deren Einsatzmöglichkeiten für Winzergenossenschaften. In diesem Kontext sind wir durch die Einführung eines Beispiels, welches die Zusammenarbeit einer Winzergenossenschaft mit einem bekannten Winzers zur Erstellung eines Premiumweins bei Aldi Süd aufbereitet, in der Lage, die Erweiterung des Handlungsspielraums des Genossenschaftsmanagements $\mathrm{zu}$ diskutieren. Mit einer kurzen Zusammenfassung beschließen wir unseren Beitrag.

\section{Genossenschaftsproblematik}

Im Genossenschaftsbereich und besonders im Bereich der Winzergenossenschaften durch starken Einfluss von Qualitätsschwankungen gibt es immer wiederkehrende Probleme. Die besondere Struktur von Genossenschaften ermöglicht es den Mitgliedern, Verhaltensweisen zu etablieren, die in anderen Geschäftsformen weniger gibt. Da von der Gesamtqualität des Produktes alle zu gleichen Teilen profitieren, obwohl die Kosten, die bei der Traubenproduktion entstehen, individuell unterschiedlich sind und individuell getragen werden müssen (Dilger, 2005), wird opportunistisches Verhalten ermöglicht. Halten sich einzelne Winzer bei ihren Anstrengungen zurück, können sie Kosten sparen und profitieren trotzdem vom allgemein höheren Qualitätsnivieau. Diese Trittbrettfahrerproblematik nimmt mit zunehmender Mitgliederzahl zu (Frick, 2004). Um dies zu vermeiden, werden unterschiedliche Entlohnungssysteme genutzt. Je stärker die Entlohnung abhängig von der individuell angelieferten Produktion ist, desto weniger schwerwiegend ist die Trittbrettfahrerproblematik (Pennerstorfer und Weiss, 2013). Generell ist heute in Winzergenossenschaften eine enge Beziehung zwischen Entlohnung und Qualität der angelieferten Ware vorzufinden (Alger et al., 2007). Dennoch werden
Genossenschaftsmitglieder nach der Menge entlohnt, so lange sie eine Mindestqualität einhalten. Sie werden sich daher im Rahmen einer bestimmten Qualitätsvorgabe am mengenmäßig oberen Rand bewegen (Dilger, 2005).

Des Weiteren können Trauben, welche zur Herstellung von qualitativ besonders hochwertigen Weinen benötigt werden, zudem unabhängig von der Genossenschaft vertrieben werden. Letztendlich qualitativ herausragende Winzer können aus der Genossenschaft austreten und sich direkt selbstständig machen (Dilger, 2005). Insbesondere Genossenschaften, die eine Teilablieferung der Trauben offiziell erlauben oder faktisch tolerieren, haben das Problem, dass die nicht genau abschätzen können, welche Mengen sie an den Markt geben können (Frick, 2004). Zudem werden die Mengen stark durch die Witterung beeinflusst. Insbesondere können Probleme dann auftreten, wenn sich die Messung er Qualitätsindikatoren als schwierig oder teuer darstellt (Frick, 2004). Indikatoren für Weinqualität ergeben sich aus auf der einen Seite durch Messungen, die während der Anlieferung vorgenommen werden, sowie auf der anderen Seite durch bestimmte Produktionsmethoden, die während der Wachstumszeit durchgeführt werden. Diese Informationen können nur während des Jahres durch Kontrollen gewonnen werden und sind somit nur schwer kontrollierbar (Goodhue et al., 2003). Hieraus ergibt sich ein weiterer Handlungsspielraum für opportunistisches Verhalten.

\section{Einflussstrategien}

Besonders für die Geschäftsführung von Genossenschaften ist die Ausgestaltung der Kooperation zwischen Genossenschaftsmitgliedern und der Genossenschaft bzw. ihr als Geschäftsführung von besonderer Bedeutung. Anders als in anderen Supply Chains ist eine Genossenschaft nicht ausschließlich Teil eines vertikal koordinierten Netzwerkes. Die Eigentumsstruktur der Genossenschaft führt dazu, dass die Geschäftsführung den Mitgliedern nicht nur Anweisungen geben kann sondern umgekehrt von ihnen als Eigentümern auch Anweisungen empfangen kann. Zudem wird die Geschäftsführung Interesse daran haben, die eigene Tätigkeit langfristig auszuüben und nicht jederzeit entlassen zu werden.

Um die Ziele, möglichst hohen geschäftlichen Erfolg bei gleichzeitiger möglichst positiver Beziehung zwischen sich und den Genossenschaftsmitgliedern zu erreichen, muss die Geschäftsführung die verschiedenen Strategien Einfluss auszuüben kennen und erfolgreich anwenden. Einflussstrategien sind die verschiedenen Möglichkeiten, Macht auszuüben, die von Personen genutzt werden können bei dem Versuch andere Mitglieder der Lieferkette zu beeinflussen (Frazier und Summers, 1986). Die Basis der verschiedenen Möglichkeiten, Einfluss auszuüben, sind die Grundlagen sozialer Macht. Es gibt diverse verschiede Klassifikationen (vgl. z.B. Simon, 1957; Tedeschi und Lindskold, 1976; Mintzberg, 1983), von denen die Klassifikation von French und Raven (1959) mit der Erweiterung von Raven (1965) als die am meisten verwendete (Mintzberg, 1983) gilt:

Soziale Einflussnahme ist definiert als gezielte Änderung der Wahrnehmung, der Einstellung und des 
Verhaltens einer Person. Bei der Betrachtung der Einflussstrategien muss die Beziehung zwischen zwei Personen betrachtet werden, auf welcher der Einfluss einer Person auf die andere beruht. French und Raven unterscheiden dabei ob die Einflussnahme dauerhaft von einer Person abhängig ist oder nicht und falls ja, ob die Einflussnahme nur durch Kontrolle aufrecht gehalten oder auch ohne eine Verhaltensänderung erreicht wird (French und Raven, 1959).

Einfluss durch Belohnung hängt von der Fähigkeit $\mathrm{ab}, \mathrm{zu}$ belohnen oder Belohnungen zu versprechen. Die Belohnung kann sowohl ein positiver Anreiz sein als auch die Verringerung von negativen Einflüssen. Die Stärke des Einflusses hängt davon $\mathrm{ab}$, wie hoch die Belohnung ist und wie wahrscheinlich es ist, dass sie eintrifft (French und Raven, 1959).

Einfluss durch Zwang ist die Fähigkeit zur Androhung von Bestrafung bei Nichterfüllen des Ziels. Dessen Stärke ist abhängig von der Bestrafung, beziehungsweise der Höhe der Strafe und der Wahrscheinlichkeit, dass das Ziel erreicht wird und die Strafe nicht eintritt (French und Raven, 1959).

Einfluss durch Belohnung und Zwang hängen beide von der Person ab und der Gegenstand muss kontrollierbar sein. Einfluss durch Legitimation, Identifikation und Expertenwissen hängen ebenfalls von der Person $a b$, müssen sich aber nicht auf kontrollierbare Verhaltensänderungen beziehen, das heißt, er funktioniert auch, wenn der Beeinflusste weiß, dass der Gegenstand nicht bemerkt wird (Raven, 1974).

Legitimer Einfluss /Einfluss durch Legitimation entsteht durch die Einstellung, die jemanden dazu bewegt, den Einfluss zu akzeptieren, den eine Person ausübt. Das können verinnerlichte Werte wie Respekt vor dem Alter oder einem Gebrechen sein, oder das Anerkennen von Autorität in einer sozialen Struktur, die auch an andere Personen vergeben werden kann. Die Stärke des Einflusses ist abhängig vom Grad der Überzeugung der Person, wieviel Recht der Einfluss Ausübende hat, diese Position inne zu haben (French und Raven, 1959).

Einfluss durch Identifikation und Vorbildcharakter liegt die Identifikation mit einer Person oder Gruppe zugrunde. Die Attraktivität des Einflussausübenden weckt den Wunsch nach Verbundenheit und Teilhabe und führt zur Kopie von wünschenswerten Eigenschaften und Zielen. Je größer die Attraktivität der Gruppe/ Person, desto größer wird der Wunsch nach Identifikation sein und somit die Möglichkeit, Einfluss zu nehmen (French und Raven, 1959).

Einfluss durch Expertenwissen entsteht durch für die Personen wertvolles Wissen. Der Einflussbereich ist beschränkt auf den Bereich des Expertenwissens. Die Stärke des Einflusses variiert mit dem Wissen im Vergleich zum Standard und zum eigenen Wissen (French und Raven, 1959).

Einfluss durch Informationsvorsprung ist die Möglichkeit, andere zu beeinflussen durch die Kommunikation von Informationen und nicht nur deren bloßes Vorhandensein. Durch gezieltes Kommunizieren von wahren oder falschen Informationen lässt sich der Empfänger beeinflussen (Raven und Kruglanski, 1970).

\section{Wirkung der Einflussstrategien der Genossenschaftsführung auf die Mitgliederbeziehung}

Einflussnahme durch Zwang wird in Verbindung gebracht mit Konflikten innerhalb der Unternehmen der Lieferkette (Hunt und Nevin, 1974; Lusch, 1976). Untersuchungen über den Einfluss von Zwang auf kooperative Beziehungen haben gezeigt, dass die bestrafenden und aggressiven Aspekte dieser Form sich negativ auf die Beziehung auswirken (Hanf et al., 2012; Belaya, 1999). Generell zeigt sich, dass Anwendung von Zwang sich negativ auf die Zufriedenheit auswirkt (Shelby und Hunt, 1974). Das Nutzen von Zwang reduziert das Vertrauen und Engagement der anderen Partei (Kumar et al., 1998).

Einflussnahme durch Belohnung kann sowohl positive wie auch negative Auswirkungen auf Kooperation haben. Angemessene Belohnungen für erbrachte Leistungen werden von Lieferanten positiv aufgenommen (Gaski, 1986). Überdurchschnittliche hohe oder zu häufige Belohnungen werden dagegen als nicht seriös aufgefasst (Skinner et al. 1992). Untersuchungen zeigen, dass übermäßige Belohnungen sich nicht positiv auf die langfristige Kooperationswilligkeit auswirken (Hanf und Belaya, 2012) sowie zu Kapitulation und dem Wunsch führen, die Beziehung zu beenden (Dapiran und Hogarth-Scott, 2003).

In Genossenschaften wird die Möglichkeit, Zwang durch Androhung von harten Bestrafungen/Sanktionen auszuüben, zusätzlich durch die Eigentümerstruktur erschwert. Durch verschiedene qualitative und quantitative Modelle gibt es Möglichkeiten, bei der Auszahlung von Traubengeld finanzielle Anreize und auch Sanktionen in einem System zu etablieren. Neben finanziellen Sanktionen gibt es auch die Möglichkeiten der AusschlussSelektion (Ringle, 2005), bei der einzelne Genossenschaftsmitglieder unfreiwillig aus der Genossenschaft entfernt werden. Solche Verfahren werden vermutlich schwierig und eher nur in extremen Fällen durchzusetzen sein, da kein Genossenschaftsmitglied für den Ausschluss eines Genossen stimmen wird, wenn dieser eine durchschnittliche Leistung zeigt und man somit auch den eigenen Ausschluss riskiert.

Beeinflussung durch Expertenwissen in Form von Weiterbildung oder Anleitung wird als Anreiz betrachtet, sich Kooperationen anzuschließen und zu öffnen (Davies et al., 2004). Diese wirken sich äußerst positiv und langfristig auf die Kooperativität der Beteiligten aus (Belaya, 1999; Dapiran und Hogarth-Scott, 2003).

Für Genossenschaften bietet sich dieses Instrument an, da zum Teil ohnehin vorhandene Ressourcen genutzt werden können. Die Genossenschaftsführung kann sich in Bereichen, in denen sie Fachwissen besitzt, darauf berufen. Ebenso kann der Kellermeister in die Mitgliedsbetriebe gehen und Beratung anbieten. Hierbei ist entscheidend, wie breit und tief die Expertise ist.

Der Einfluss durch Informationsvorsprung setzt voraus, dass der Beeinflusste die gegebenen Informationen verinnerlicht und sich zu eigen macht (Raven, 1974). Es ist davon auszugehen, dass diese Art der Einflussnahme 
Kooperation positiv beeinflusst (Dapiran und HogarthScott, 2003), allerdings zumindest einen gewissen Grad an vorhandener Kooperation voraussetzt, da sonst die Informationen nicht angenommen werden. Studien zeigen, dass Nutzen von Informationen Kooperationen positiv beeinflusst, da aktuelle und hochwertige Informationen wichtig für den Erfolg sind (Belaya, 1999). Diese Form der Einflussnahme ist für die Genossenschaftsführung interessant, da das jeweilige Ziel bei positiver Beeinflussung der Kooperation erreicht werden kann.

Einflussnahme durch Legitimation kann negative Auswirkungen auf Kooperation bewirken (Belaya, 1999). Bei Nutzung dieser Art des Einflusses wird angenommen, dass der Einfluss Ausübende unwillig ist sich anzupassen (Frazier und Summers, 1986). Unter Umständen kann das Nutzen oder Androhen von legitimen Maßnahmen wie Zwang wahrgenommen werden (Shelby und Hunt, 1974). Je stärker die Akzeptanz der Legitimation ist, desto leichter fällt es, die Einflussnahme zu akzeptieren und desto größer ist die Attraktivität der ausübenden Person (Raven und French, 1958). Aufgrund der unter Umständen unklaren Situation, wer in Genossenschaften tatsächlich das Sagen hat (Geschäftsführung oder Eigentümer), ist diese Strategie schwierig anzuwenden. Die zu erwartenden negativen Auswirkungen auf die Kooperation lassen diese Möglichkeit nicht als gute Option erscheinen.

Einfluss durch Vorbildcharakter dagegen zeigt positive Auswirkungen auf Kooperation und Zufriedenheit innerhalb von Kooperationen (Lee und Low, 2008; Belaya, 1999). Der Wunsch nach Teilhabe an und Identifikation mit einer Gruppe oder Person (French und Raven, 1959) zeigt, dass Interaktion gewünscht ist. Diese Option wird innerhalb von Genossenschaften allerdings schwer zu nutzen sein. Hier ist eher zu erwarten, dass erfolgreiche Genossenschaften sie nutzen, um mit ihrer Außenwirkung neue Mitglieder anzuziehen, bzw. innerhalb der Genossenschaft den Zusammenhalt zu stärken. Möglich wäre es, einzelne besonders herausragende Mitglieder zu präsentieren und als Vorbild für andere aufzubauen.

\section{Erweiterung der Einflussspielraums durch Zusammenarbeit mit herausragenden Winzern}

Im Folgenden soll die Kooperation zwischen einem erfolgreichen Winzer und Genossenschaften beschrieben werden sowie die Möglichkeiten, die sich in solch einem Ansatz für Genossenschaften eröffnen könnten.

Seit 2008 ist bei Aldi Süd die Edition Fritz Keller zu finden. Zwei Weine, ein Weiß- und ein Spätburgunder, werden im Preisbereich von sechs bis sieben Euro angeboten. Der erste Jahrgang 2007 wurde noch von 430 Winzern auf 130 ha produziert, der Folgejahrgang von 750 Winzern mit über 300 ha (LZ, 2009). Der Großteil der benötigten Menge kommt aus Winzergenossenschaften (SüDKURIER, 2007). Circa die Hälfte der Produzenten gehören zum Badischen Winzerkeller (LZ, 2009).

Das Projekt ist eine Kooperation von 16 Winzergenossenschaften, Weingütern und Privatkellereien mit der Vitis Projekt GmbH. Das ist ein Joint Venture zwischen Fritz Keller und Franz Frickenstein, Mitinhaber von
C.A. Warren. Bei der Weinbereitung werden besondere Qualitätsmaßstäbe angesetzt. Statt den erlaubten 90 Litern pro $\mathrm{Ar}$ dürfen beteiligte Winzer nicht mehr als 55 Liter je Ar (durchschnittliche Erträge liegen in Baden 2009-2012 zwischen 69 und 89 Liter je Ar [DWI, 2014]) ernten (Badische Zeitung, 2009). Das Mindestalter der Reben muss bei 10 Jahren liegen. Zusätzliche Vorgaben sind Grün- und Handlese. Für das Projekt sind 40 Mitarbeiter für die Qualitätskontrolle der einzelnen Betriebe zuständig. (LZ, 2009).

Fritz Keller vom Weingut Franz Keller Schwarzer Adler ist in der Weinbranche ein bekannter Name. Es ist zwar davon auszugehen, dass nicht jeder Aldi-Kunde mit diesem Namen etwas verbindet und der Verkaufserfolg der Weine nicht nur dem Markennamen geschuldet ist. Gerade in Baden kennen ihn jedoch vermutlich viele, da er nicht nur als erfolgreicher Winzer, Hotelier, Gastronom und Weinhändler in Erscheinung tritt, sondern auch als Vorsitzender des Fußballvereins SC Freiburg.

Durch die Zusammenarbeit mit einem so bekannten Winzer bietet sich für die Genossenschaftsführung eine massive Erweiterung des Spielraums von Einflussstrategien. Die sich eher negativ auf die Kooperation auswirkenden Einflussmöglichkeiten der Bestrafung und Sanktion können auf den Druck des Winzers geschoben werden. Die Kooperation innerhalb der Genossenschaft bleibt bestehen und die angestrebten Ziele werden nach Möglichkeit erreicht.

Einfluss durch Expertenwissen wird dem sehr erfolgreichen Kooperationspartner eine stärkere Durchschlagskraft geben lassen als der Geschäftsführung, da von diesem angenommen wird, dass er über zusätzliches Wissen verfügt, welches ihn so erfolgreich macht.

Dem Einfluss durch Identifikation und Vorbildcharakter wird durch die Zusammenarbeit eine starke Wirkung zukommen. Es kann sich eine Gruppe innerhalb der Gruppe bilden, die eine starke Außenwirkung hat. Neben den "normalen" Genossenschaftswinzern gibt es die besondere Gruppe, die auserwählt wurde und leisten kann, was gefordert wird. Dies wird Außenstehende veranlassen sich anzustrengen, um Teil der Gruppe werden zu können.

Weiter wird innerhalb der Gruppe die Möglichkeit geschaffen, jemanden hinauszuwerfen (zu sanktionieren), ohne dass die negativen Auswirkungen auf die Geschäftsführung projiziert werden und ohne dass jemand direkt aus der Genossenschaft entfernt werden muss. Somit muss dafür auch keine Mehrheit in der GenossenschaftsMitgliederversammlung gefunden werden. Auch schafft schon das bloße Vorhandensein dieser Option eine Bedrohung, da der Ausschluss aus der attraktiven Gruppe auch eine soziale Komponente besitzt. In einem eng abgegrenzten Gebiet würde sich so ein Vorgang schwer verheimlichen lassen.

Einfluss durch Legitimation wird in diesem Modell auch in stärkerem Umfang verwendet werden können, als dies in normalen genossenschaftlichen Geschäftsverhältnissen möglich ist. Durch eine übergeordnete Instanz fällt die Problematik der ungeklärten Hierarchie weg und zudem wird einer erfolgreichen, durch lokale Bekanntheit ausgewiesenen Autorität eher eine übergeordnete Stellung zugebilligt. 


\section{Zusammenfassung}

Für Genossenschaften ist es eine Herausforderung, Mengen, Qualitäten und opportunistisches Verhalten zu kontrollieren und koordinieren. Viele dort aufzufindende Problematiken entstehen durch Interessenkonflikte und mangelnde Motivation.

Bei der Produktion großer hochwertiger Mengen kommen diese Kooperationsprobleme besonders zum Tragen. Es wurden die verschiedenen Einflussstrategien wie Zwang, Belohnung, Legitimation, Identifikation, Expertenwissen und Informationsvorsprung vorgestellt und unter Berücksichtigung der besonderen Genossenschaftsstruktur in ihrer Wirkung auf die Mitgliederbeziehung untersucht.

Im Weiteren wurde anhand des Beispiels der Edition Fritz Keller die Zusammenarbeit zwischen einem prominenten Winzer und Winzergenossenschaften gezeigt, wie der Spielraum der sozialen Einflussnahme erweitert werden kann.

Dieses Beispiel zeigt, dass Genossenschaften mit ihren vorstehend dargestellten Problemen durch innovative Lösungen ihre Nachteile ausgleichen können. Die Edition Fritz Keller ist sicherlich ein Paradebeispiel, das nicht ohne weiteres reproduzierbar und auch nicht eins zu eins umzusetzen ist, da hier mehrere Unternehmen und Genossenschaften zusammenarbeiten. Dennoch ist auch für einzelne Unternehmen die Zusammenarbeit mit erfolgreichen lokalen oder regionalen Winzern eine Option, hochwertige Editionen $\mathrm{zu}$ lancieren und unternehmensintern Qualitätswettbewerb zu fördern.

\section{Literaturverzeichnis}

Alger, M., A. Fritsch und R. Reichel, 2007:Winzergenossenschaften im Wettbewerb; in: ZfgG; 2007; S. 167-177

Badische Zeitung, 2007a: "Chance für Baden" - TopWinzer Fritz Keller über seine Kooperation mit Aldi; in: Badische Zeitung, 06.05.2007

Badische Zeitung, 2007b: Teurer Wein beim Billiganbieter - Der Wein, den der Kaiserstühle Spitzengastronom und Weinproduzent Fritz Keller für den Discounter schaffen will, soll den Ruf des badischen Weins fördern; in: Badische Zeitung, 08.05.2007

Badische Zeitung, 2008: Fritz Keller bei Aldi; in: Badische Zeitung, 20.04.2008

Badische Zeitung, 2009: Eine ausgegorene Idee - Fritz Kellers Aldi-Weine bringen den hiesigen Winzern offenbar zusätzlichen Absatz; in Badische Zeitung, 10.03.2009

Belaya, V., 2012: Influence Strategies in Suply Chains and Networks - Study of Russian Agri-Food Business; Dissertation; Martin-Luther-Universität Halle-Wittenberg

Cook, L und C. Iliopoulos, 1999: Beginning to Inform the Theory of the Cooperative Firm: Emergence of the New Generation Cooperative; in: The Finnish Journal of Business EcoNrmics; Nr.4/99; S. 525-535

Cooper, M., D. Lambert, und J. Pagh, 1997: Supply chain management: more than a new name for logistics, in:
International Journal of Logistics Management, 8, Nr. 2; S. 1-14

Dapiran, G und S. Hogarth-Scott, 2003: Are co-operation and trust being confused with poal Journal of Retail \& Distribution Management; 31, Nr. 5; S. 256-267wer? An analysis of food retailing in Australia and the UK; in: International Journal of Retail \& Distribution Management; Bd. 31, Nr. 5; S. 256-267

Davies, B., K. Blackstock, K. Brown und P. Shannon, 2004: Challenges in creating Local agrienvironmental cooperation action amongst farmers and other stakeholders; Final Report of Scottish Executive Environment and Rural Affairs Department; Aberdeen, UK

Dilger, A. 2005: Prinzipal-Agenten-Probleme im deutschen Weinbau; in: ZfgG; S. 179-189

DWI, 2014: Deutsches Weininstitut, http://www . deutscheweine.de/icc/Internet-DE/med/4fd/ 4fd50473-eaa2-3e21-23f3-74c25f440fd3, 11111111-1111-1111-1111-111111111111.pdf; abgerufen am 12.02.2014

Ellram, L. und M. Cooper, 1990: Supply Chain Management, Partnership, and theShipper - Third Party Relationship; in: The International Journal of Logistics Management; 1, Nr. 2; S. 1-10

Frazier, G. und J. Summers, 1986: Perceptions of interfirm power and its use within a franchise channel of distribution; in: Journal of Marketing Research (JMR), 23, Nr. 2; S. 169-176

French, J. und B. Raven, 1959: The Bases of Social Power; in: Cartwright, D. (Hrsg); Studies in Social Power; University of Michigan Press; Ann Arbor, MI; S. 150167

Frick, B., 2004: Does Ownership Matter? Empirical Evidence from the German Wine Industry; in: Kyklos; John Wiley \& Sons Ltd; Hoboken, NJ; S. 357-386

Gaski, J., 1986: The theory of power and conflict in channels of distribution; in: Journal of Marketing; 48. Nr. 3, S. 9-29

Goodhue, R., D. Heien, H. Lee und D. Sumner, 2003: Contracts and quality in the California winegrape industry; in: Review of Industrial Organization; 23; 267-282

Gulati, R., P.R. Lawrence and P. Puranam, 2005: Adaptation in vertical relationships: Beyond incentive conflicts; in: Strategic Management Journal, 26, S. 415440

Halldorsson, A., H. Kotzab, J. Mikkola und T. SkjøttLarsen, 2007: Complementary theories to supply chain management; in: Supply chain management: An international journal, 12, Nr. 4; S. 284-296

Hanf, J. und K. Dautzenberg, 2006: A theoretical framework of chain management; in: Journal on Chain und Network Science; 6; S. 79-94

Hanf, J. und V. Belaya, 2012: Managing Russian agrifood supply chain networks with power; in: Journal on Chain and Network Science; S. 215-230

Hanf, J., V. Belaya und E. Schweickert, 2012: Power Play in the German Wine Business: Are German Wine 
Co-operatives able to Use their Power to manage their Business Relationships?; in: Journal of Economics and Behavioral Studies; 4; Nr. 4; S. 227-238

Hanf, J. und E. Schweickert, 2014: Cooperatives in the balance between retail and member interests: The challenges of the German cooperative sector, Journal of Wine Research. 25, Nr. 1; S. 1-13

Hunt, S. und J. Nevin, 1974: Power in a Channel of Distribution: Sources and Consequences; in: Journal of Marketing Research; 11, Nr. 2; S. 186-193

Kumar, N., L. Scheer und J.-B. Steenkamp, 1998: Interdependence, punitive capability, and the reciprocation of punitive actions in channel relationships; in: Journal of Marketing Research, 35, S. 225- 235

Lee, K. und G. Low, 2008: The Exercise of Social Power and the Effect of Ethnicity: Evidence from Malaysian's Industrial Companies; in: International Business Research, 1, Nr. 2, S. 53-65

Lusch, R., 1976: Sources of Power: Their Impact on Intrachannel Conflict; in: Journal of Marketing Research (JMR); 13, Nr. 4

LZ., 2009 Discounter punkten beim Wein; in: Lebensmittelzeitung, 07.08.2009; S. 20

McEvily, B., V. Perrone und A. Zaheer, 2003: Trust as an Organizing Principle; in: Organization Science; 14, Nr. 1; S. 91-103

Mintzberg, H., 1983: Power in and around organizations (142). Englewood Cliffs, NJ: Prentice-Hall

Pennerstorfer, D. und C. Weiss, 2013: Product quality in the agri-food chain: do cooperatives offer highquality wine?; in: European Review of Agricultural Economics; 40 (1); Den Haag; S. 143-162
Preckel, P., M. Boehlje, A. Gray und s. Kim, 2004: Vertically Aligned vs. Open Market Coordination: Dominance or Co-Existence?; Paper presented at the Annual Meeting of American Agricultural Economics Association; Denver, USA;1.-4. August 2004

Raven, B., 1974: The Comparative Analysis of Power and Power Preference; in : J. Tedeischi (Hrsg.); Social Power and Political Influence; Transaction publishers, New Brunswick; S. 172-200

Raven, B. und J.French, 1958: Legitimate power, coercive power, and observability in social influence; in: Sociometry; 21; S. 83-97

Raven, B. und A. Kruglanski, 1970: Control and Power; in: P. Swingle (Hrsg.); The Structure of Conflict; Academic; New York; S. 69-109

Ringle, G., 2005: Selektive Mitgliederaufnahme in GeNrssenschaften aus heutiger Sicht; in: ZfgG; S. 123-135

Simon, H. (1957): Authority, in: Arensberg, Barkin, Chalmers, Wilensky, Worthy, Dennis (Hrsg); Research in Industrial Relations; New York; S. 103-115

Skinner, S., J. Gassenheimer und S. Kelley, 1992: Cooperation in Supplier Dealer Relations; Journal of Retailing; 68, Nr. 2, S. 174-193

Südkurier, 2007: Discounter verpflichtet Edelwinzer/Fritz Keller entwirft und produziert hochpreisige Qualitätsweine für Aldi-Süd; in: Südkurier, 11.05.2007

Tedeschi, J. und S. Lindskold, 1976: Social Psychology: Interdependence, Interaction and Influence; John Wiley \& Sons; Oxford

Vitis Projekt GmbH, 2014: http://www.vitisprojekt.de; abgerufen am 22.01.2014 